\title{
Tuning pure out-of-plane piezoelectric effect of penta-graphene: a first-principle study
}

\author{
San-Dong Guo \\ School of Electronic Engineering, Xi'an University of Posts and Telecommunications, Xi'an 710121, China
}

\begin{abstract}
For two-dimensional (2D) materials, a pure large out-of-plane piezoelectric response, compatible with the nowadays bottom/top gate technologies, is highly desired. In this work, the piezoelectric properties of penta-graphene (CCC) monolayer are studied with pure out-of-plane piezoelectric effect by density functional theory (DFT). However, the $d_{36}$ is very small, and only $-0.065 \mathrm{pm} / \mathrm{V}$. Two strategies are proposed to enhance piezoelectric properties of CCC monolayer. Firstly, both biaxial and uniaxial strains are applied, but the enhancement is very small, and at $-2 \%$ biaxial $(-4 \%$ uniaxial) strain, the $d_{36}$ is increased only by $3.1 \%(13.9 \%)$. Secondly, a Janus penta-monolayer $(\mathrm{CCB})$ is constructed by replacing the top $\mathrm{C}(\mathrm{B})$ atomic layer in monolayer $\mathrm{CCC}$ [pentagonal $\mathrm{CB}_{2}$ monolayer (CBB)] with B (C) atoms, which shows dynamic and mechanical stability. Fortunately, the pure out-of-plane piezoelectric effect of CCB monolayer still holds, and exhibits a band gap. The calculated $d_{31}$ and $d_{32}$ are $-0.505 \mathrm{pm} / \mathrm{V}$ and $0.273 \mathrm{pm} / \mathrm{V}$, respectively, which are very larger than $d_{36}$ of CCC monolayer. The out-of-plane piezoelectricity $d_{31}$ of CCB monolayer is obviously higher compared with many other 2D known materials. Moreover, its room-temperature electronic mobility along y direction is as high as $8865.23 \mathrm{~cm}^{2} \mathrm{~V}^{-1} \mathrm{~s}^{-1}$. Our works provide a new way to achieve pure out-of-plane piezoelectric effect, which is highly desirable for ultrathin piezoelectric devices.
\end{abstract}

PACS numbers: 71.20.-b, 77.65.-j, 72.15.Jf, 78.67.-n

Keywords: Penta-graphene, Piezoelectronics, 2D materials,

\section{INTRODUCTION}

Beyond graphene, a large amount of 2D carbon allotropes have been investigated. The CCC monolayer of them, composed entirely of carbon pentagons, could be realized experimentally with good thermodynamic stability ${ }^{1}$. Inspired from CCC monolayer, other pentagon-based 2D materials have been theoretically predicted or experimentally synthesized, such as penta$\mathrm{CB}_{2}{ }^{2}$, penta-SnX $\mathrm{X}_{2}(\mathrm{X}=\mathrm{S}, \mathrm{Se} \text {, or } \mathrm{Te})^{3}$ and penta- $\mathrm{AlN}_{2}{ }^{4}$. The mechanical behavior of monolayer CCC under multiaxial loading has been studied by DFT calculations, and the structure has lower ultimate tensile strength compared to graphene ${ }^{5}$. The phonon transport properties of CCC monolayer have been widely investigated theoretically, and the intrinsic lattice thermal conductivity is significantly reduced as compared to that of graphene ${ }^{6-8}$. The magnetic moments can be induced by an isolated hydrogen atom absorbed on CCC monolayer, which changes CCC monolayer from a semiconductor to half-metallic ${ }^{9}$. However, to the best of our knowledge, piezoelectric properties of CCC monolayer haven't been reported.

In fact, the piezoelectricity in 2D materias has attracted growing interest ${ }^{10}$ because of potential nanoscale piezoelectric applications, like sensors, actuators and energy sources. For 2D materials, the reduction in dimensionality makes their inversion symmetry disappear, which allows them to become piezoelectric. Experimentally, the monolayer $\mathrm{MoS}_{2}$ with the $2 \mathrm{H}$ phase has been proved to be piezoelectric $\left(e_{11}=2.9 \times 10^{-10} \mathrm{C} / \mathrm{m}\right)^{11,12}$, and the existence of vertical dipoles in the Janus MoSSe monolayer has also been observed, showing an intrinsic vertical piezoelectric response ${ }^{13}$. In theory, lots of studies on piezoelectric properties related with $2 \mathrm{D}$ materials have been reported ${ }^{14-18}$, like transition metal dichalchogenides

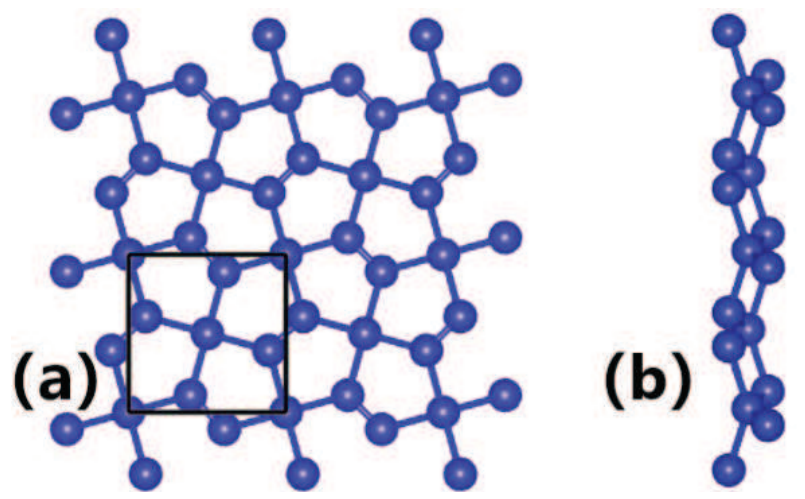

FIG. 1. (Color online)The crystal structure of pentagraphene: top view (a) and side view (b), and the primitive cell is are marked by black line.

(TMD), group IIA and IIB metal oxides, group III$\mathrm{V}$ semiconductors, group- $\mathrm{V}$ binary semiconductors and Janus TMD. It is surprising that the giant piezoelectricities in monolayer SnSe, SnS, GeSe and GeS have been predicted by the first-principle calculatios ${ }^{16}$, as high as $75-251 \mathrm{pm} / \mathrm{V}$ along the the armchair direction. Some 2D materials only exhibit an in-plane piezoelectricity, like TMD monolayers ${ }^{15}$, and an additional out-of-plane piezoelectricity has been observed in many $2 \mathrm{D}$ materials, like Janus $\mathrm{TMD}^{14}$. A pure large out-of-plane piezoelectric response is highly desired, which is compatible with the nowadays bottom/top gate technologies. However, the pure out-of-plane piezoelectric response in 2D materials is rarely reported.

In this work, the piezoelectric properties of CCC monolayer are reported by using density functional perturbation theory (DFPT) ${ }^{19}$ with generalized gradient approx- 
imation (GGA). Only out-of-plane $d_{36}$ exists for CCC monolayer, but it is very small. Both biaxial and uniaxial strains are used to tune its piezoelectric properties, but the improvement is very small. Janus monolayer can be built from symmetric sandwich structure, like MoSSe synthesized by replacing the top S atomic layer in $\mathrm{MoS}_{2}$ with Se atoms ${ }^{13}$. Inspiring from MoSSe monolayer, a Janus CCB monolayer with dynamic and mechanical stability is constructed, and the special symmetry leads to only out-of-plane piezoelectric effect. Compared with $d_{36}$ of CCC monolayer, the predicted $d_{31}$ and $d_{32}$ obviously are improved, the $d_{31}$ of which is higher compared with other many 2D known materials. Another significant advantage for CCB monolayer is a very high room-temperature electronic mobility (8865.23 $\mathrm{cm}^{2} \mathrm{~V}^{-1} \mathrm{~s}^{-1}$ ) along y direction. Therefore, our works give an experimental proposal to achieve pure out-of-plane piezoelectricity in 2D materials, and pave a way for designing piezoelectric devices compatible with the nowadays bottom/top gate technologies.

The rest of the paper is organized as follows. In the next section, we shall give our computational details and methods about piezoelectric coefficients. In the third and fourth sections, we shall present piezoelectric properties of monolayer CCC and CCB. Finally, we shall give our conclusions in the fifth section.

\section{COMPUTATIONAL DETAIL}

Within the framework of $\mathrm{DFT}^{20}$, our calculations are performed by using the VASP package ${ }^{21-23}$. The projected augmented wave (PAW) method with a kinetic cutoff energy of $500 \mathrm{eV}$ is adopted, and we use the popular GGA of Perdew, Burke and Ernzerhof (GGA-PBE) ${ }^{24}$ as the exchange-correlation potential. For all studied monolayers, a vacuum spacing of more than $16 \AA$ along the $\mathrm{z}$ direction is included to avoid interactions between two neighboring images. The total energy convergence criterion is set to $10^{-8} \mathrm{eV}$, and the Hellmann-Feynman forces on each atom are less than $0.0001 \mathrm{eV} . \AA^{-1}$. The coefficients of the elastic stiffness tensor $C_{i j}$ are calculated by using the finite difference method (FDM), and the piezoelectric stress coefficients $e_{i j}$ are calculated by DFPT method ${ }^{19}$. Within FDM and DFPT, the electronic and ionic contribution to the elastic and piezoelectric stress coefficient can be attained directly from VASP code. For $C_{i j}$ and $e_{i j}$, the Brillouin zone sampling is done using a Monkhorst-Pack mesh of $23 \times 23 \times 1$ for CCC monolayer and CBB monolayer, and $18 \times 19 \times 1$ for CCB monolayer. The $2 \mathrm{D}$ elastic coefficients $C_{i j}^{2 D}$ and piezoelectric stress coefficients $e_{i j}^{2 D}$ have been renormalized by the the length of unit cell along $\mathrm{z}$ direction $(L z)$ : $C_{i j}^{2 D}=L z C_{i j}^{3 D}$ and $e_{i j}^{2 D}=L z e_{i j}^{3 D}$.
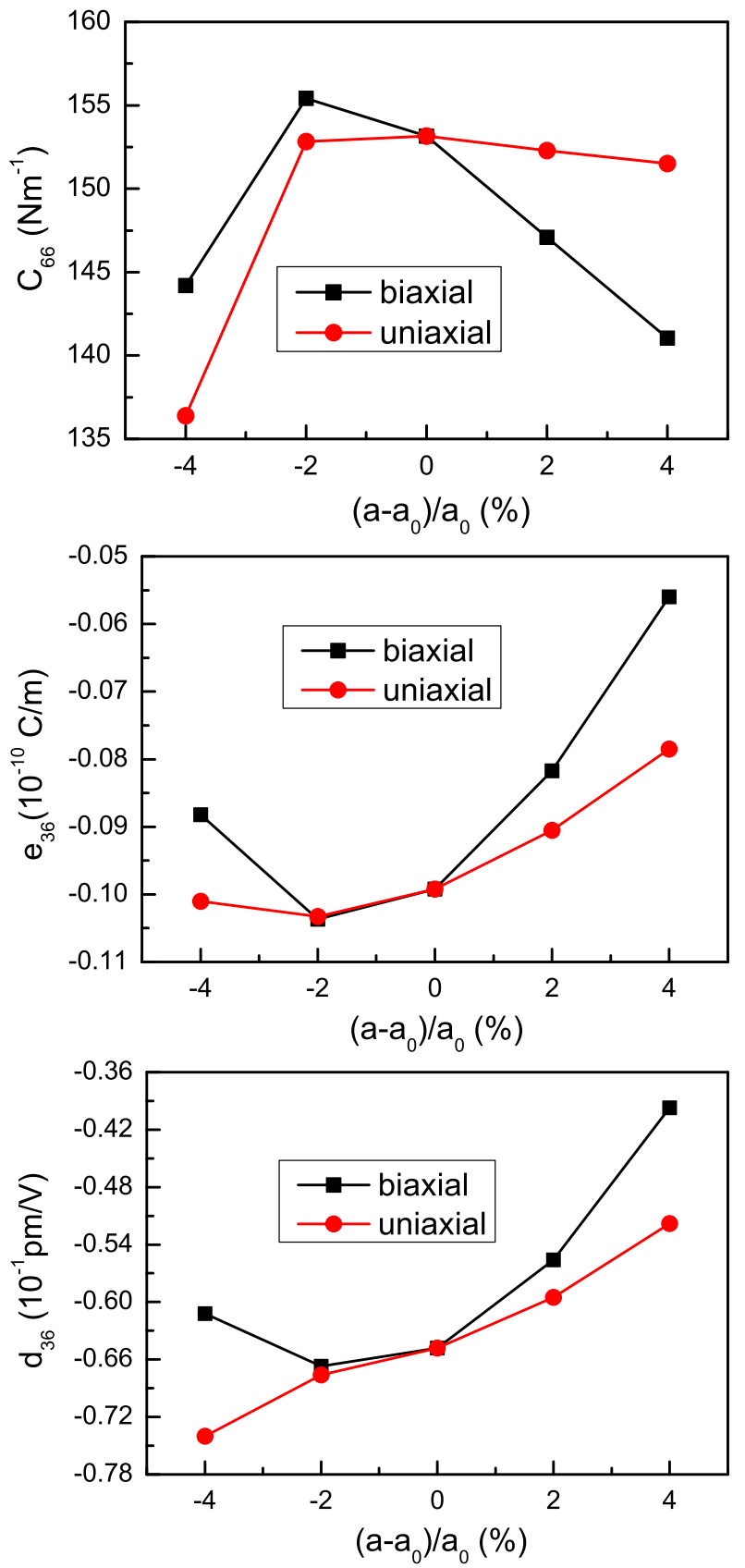

FIG. 2. (Color online) For monolayer CCC, the elastic constants $C_{66}$, piezoelectric coefficients $e_{36}$ and $d_{36}$ with the application of biaxial and uniaxial strains.

\section{PIEZOELECTRIC PROPERTIES OF CCC MONOLAYER}

The strain or stress can induce a change of polarization in noncentrosymmetric crystals, described by the thirdrank piezoelectric stress tensors $e_{i j k}$ and strain tensor $d_{i j k}$. They from the sum of ionic and electronic contributions are defined as:

$$
e_{i j k}=\frac{\partial P_{i}}{\partial \varepsilon_{j k}}=e_{i j k}^{e l c}+e_{i j k}^{i o n}
$$


TABLE I. For CCC, CCB and CBB monolayers, the lattice constants $a_{0} / b_{0}(\AA)$ and $\gamma$, the GGA gaps (eV), and the elastic constants $C_{i j}\left(\mathrm{Nm}^{-1}\right)$.

\begin{tabular}{lccccccc}
\hline \hline Name & $a_{0} / b_{0}$ & $\gamma$ & Gap & $C_{11}$ & $C_{22}$ & $C_{12}$ & $C_{66}$ \\
\hline CCC & 3.639 & 90 & 2.20 & 271.84 & 271.84 & -20.86 & 153.15 \\
\hline CCB & 3.771 & 92.107 & 0.66 & 142.36 & 193.07 & 34.40 & 61.71 \\
\hline CBB & 3.933 & 90 & 1.41 & 86.78 & 86.78 & 87.86 & 89.20 \\
\hline \hline
\end{tabular}

TABLE II. For CCC, CCB and CBB monolayers, piezoelectric coefficients $e_{i j}$ and $d_{i j}$, along with out-of-plane piezoelectric coefficients of some typical 2D materials, like $\mathrm{SbTeI}^{34}$, $\mathrm{BiTeI}^{34}, \mathrm{MoSSe}^{33}$ and MoSTe ${ }^{35}$. The unit is $10^{-10} \mathrm{C} / \mathrm{m}$ for $e_{i j}\left(\mathrm{pm} / \mathrm{V}\right.$ for $\left.d_{i j}\right)$.

\begin{tabular}{lcccccc}
\hline \hline Name & $e_{31}$ & $d_{31}$ & $e_{32}$ & $d_{32}$ & $e_{36}$ & $d_{36}$ \\
\hline \hline CCC & & & & & -0.099 & -0.065 \\
\hline CCB & -0.624 & -0.505 & 0.353 & 0.273 & & \\
\hline CBB & & & & & -0.372 & -0.418 \\
\hline \hline SbTeI & -0.13 & -0.37 & -0.13 & -0.37 & & \\
\hline BiTeI & -0.23 & -0.66 & -0.23 & -0.66 & & \\
\hline MoSSe & 0.42 & 0.29 & 0.42 & 0.29 & & \\
\hline MoSTe & 0.5 & 0.4 & 0.5 & 0.4 & & \\
\hline \hline
\end{tabular}

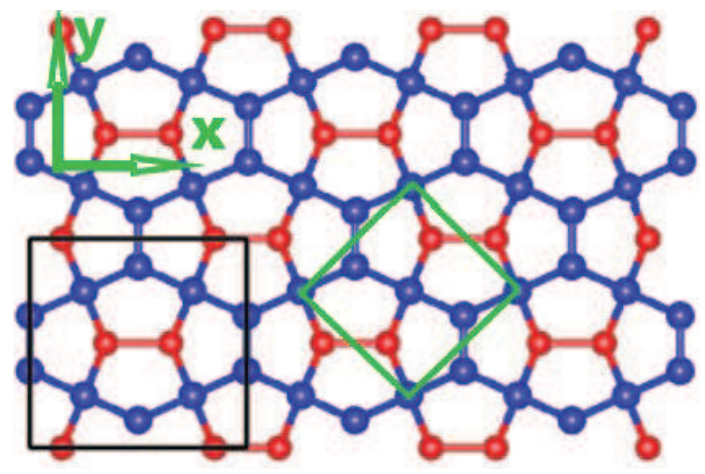

FIG. 3. (Color online) The top view of crystal structure of $\mathrm{CCB}$, and the primitive cell and rectangle supercell are marked by green and black lines, respectively.

and

$$
d_{i j k}=\frac{\partial P_{i}}{\partial \sigma_{j k}}=d_{i j k}^{e l c}+d_{i j k}^{i o n}
$$

Where $P_{i}, \varepsilon_{j k}$ and $\sigma_{j k}$ are polarization vector, strain and stress, respectively. By employing Voigt notation, for $2 \mathrm{D}$ materials with only considering in-plane strain components $^{14-18}$, the $d_{i j}$ can be derived using the relation:

$$
\left(\begin{array}{lll}
e_{11} & e_{12} & e_{16} \\
e_{21} & e_{22} & e_{26} \\
e_{31} & e_{32} & e_{36}
\end{array}\right)=\left(\begin{array}{lll}
d_{11} & d_{12} & d_{16} \\
d_{21} & d_{22} & d_{26} \\
d_{31} & d_{32} & d_{36}
\end{array}\right)\left(\begin{array}{lll}
C_{11} & C_{12} & C_{16} \\
C_{21} & C_{22} & C_{26} \\
C_{61} & C_{62} & C_{66}
\end{array}\right)
$$

Where the $C_{i j}$ is elastic tensor, which can be calculated by FDM, and the $e_{i j}$ can be attained by DFPT. The CCC monolayer has $P \overline{4} 2_{1} m$ symmetry (space group number 113), and the corresponding point group $\overline{4} 2 m$ makes Equation 3 become:

$$
\left(\begin{array}{ccc}
0 & 0 & 0 \\
0 & 0 & 0 \\
0 & 0 & e_{36}
\end{array}\right)=\left(\begin{array}{ccc}
0 & 0 & 0 \\
0 & 0 & 0 \\
0 & 0 & d_{36}
\end{array}\right)\left(\begin{array}{ccc}
C_{11} & C_{12} & 0 \\
C_{12} & C_{11} & 0 \\
0 & 0 & C_{66}
\end{array}\right)
$$

Here, the $d_{36}$ is derived by Equation 4 :

$$
d_{36}=\frac{e_{36}}{C_{66}}
$$

It is clearly seen that only out-of-plane piezoelectric effect can be observed.

The penta-graphene can be considered as a sandwich structure, with the 4-coordinated $\mathrm{C}$ atoms sandwiched between the 3-coordinated atoms, and the schematic structure is plotted in Figure 1. Firstly, the lattice constants of CCC are optimized ( $a=b=3.639 \AA)$, which is very close to previous theoretical values ${ }^{1}$. The elastic stiffness coefficients $C_{i j}$ and piezoelectric stress tensors $e_{i j}$ are calculated, and then $d_{i j}$ can be attained. By using FDM, we obtain $C_{11}=271.84 \mathrm{Nm}^{-1} C_{12}=-20.86 \mathrm{Nm}^{-1}$ and $C_{66}=153.15 \mathrm{Nm}^{-1}$. The calculated $C_{i j}$ agree well with previous ones ${ }^{1}$. The $C_{12}$ is negative, which means a negative Poisson's ratio. These related data are listed Table I. For 2D materials, a pure out-of-plane piezoelectric response, compatible with the nowadays bottom/top gate technologies, is highly desired. For CCC monolayer, only out-of-plane piezoelectric response exits, but the predicted piezoelectric coefficient $d_{36}$ is very small, and the calculated value: $e_{36}=-0.099 \times 10^{-10} \mathrm{C} / \mathrm{m}$ and $d_{36}=-0.065 \mathrm{pm} / \mathrm{V}$. Some strategies should be applied to improve piezoelectric effect of CCC monolayer, and that only out-of-plane piezoelectric response holds.

It has been proved that strain can effectively improve the piezoelectric response of $2 \mathrm{D}$ materials, such as $\mathrm{MoS}_{2}{ }^{25}, \mathrm{AsP}^{26}, \mathrm{SnSe}^{26}$ and Janus TMD monolayers ${ }^{27}$. For example, the $d_{22}$ of SnSe monolayer at $-3.5 \%$ strain along the armchair direction is up to $628.8 \mathrm{pm} / \mathrm{V}$ from unstrained $175.3 \mathrm{pm} / \mathrm{V}^{26}$. Here, the small both biaxial and uniaxial strain ( $-4 \%$ to $4 \%$ ) effects on piezoelectric properties of monolayer CCC are investigated. The elastic constants $C_{66}$, piezoelectric coefficients $e_{36}$ and $d_{36}$ as a function of strain are plotted in Figure 2. With both biaxial and uniaxial strains changing from $-4 \%$ to $4 \%$, both 
$C_{66}$ and $e_{36}$ show a non-monotonic behavior. It is found that, according to Equation 5, the compressive strain is in favour of improving piezoelectric response due to reducing $C_{66}$ and improving $e_{36}$ (absolute value). However, the enhancement is very small, and at $-2 \%$ biaxial $(-4 \%$ uniaxial) strain, the $d_{36}$ becomes -0.067 (-0.074) pm/V from unstrained $-0.065 \mathrm{pm} / \mathrm{V}$, increased only by $3.1 \%$ $(13.9 \%)$.

\section{PIEZOELECTRIC PROPERTIES OF CCB MONOLAYER}

A pentagonal CBB monolayer has also been predicted $^{2}$, which can also be viewed as a B-C-B sandwich trilayer. In other words, the $\mathrm{C}$ atoms of the first and third layers of penta-graphene are replaced by $\mathrm{B}$ atoms. The $P \overline{4} 2{ }_{1} m$ symmetry still holds for CBB monolayer, and our optimized lattice constants $a=b=3.933 \AA$, which agrees well with previous ones ${ }^{2}$. The calculated piezoelectric coefficients are $e_{36}=-0.372 \times 10^{-10} \mathrm{C} / \mathrm{m}$ and $d_{36}=-0.418 \mathrm{pm} / \mathrm{V}$, which are very larger than ones of CCC monolayer. However, our calculated elastic stiffness coefficients $\left(C_{11}=86.78 \mathrm{Nm}^{-1}, C_{12}=87.86 \mathrm{Nm}^{-1}\right.$ and $C_{66}=89.20 \mathrm{Nm}^{-1}$ ) violate the Born criteria of mechanical stability $^{28,29}$ :

$$
C_{11} C_{22}-C_{12}^{2}>0 \text { and } C_{66}>0
$$

Experimentally, Janus TMD monolayer MoSSe with sandwiched S-Mo-Se structure has been synthesized by replacing the top $\mathrm{S}$ atomic layer in $\mathrm{MoS}_{2}$ with Se atoms ${ }^{13}$. Thus, it is possible to build CCB monolayer by replacing the top $\mathrm{C}(\mathrm{B})$ atomic layer in monolayer $\mathrm{CCC}(\mathrm{CBB})$ with B (C) atoms. The schematic structure of CCB monolayer is shown in Figure 3. The symmetry of CCB monolayer reduces to $\mathrm{Cmm} 2$ (space group number 35 ), and the corresponding point group is $m m 2$. The Equation 3 changes into:

$$
\left(\begin{array}{ccc}
0 & 0 & 0 \\
0 & 0 & 0 \\
e_{31} & e_{32} & 0
\end{array}\right)=\left(\begin{array}{ccc}
0 & 0 & 0 \\
0 & 0 & 0 \\
d_{31} & d_{32} & 0
\end{array}\right)\left(\begin{array}{ccc}
C_{11} & C_{12} & 0 \\
C_{12} & C_{22} & 0 \\
0 & 0 & C_{66}
\end{array}\right)
$$

Here, the $d_{31}$ and $d_{32}$ are derived by Equation 7:

$$
\begin{aligned}
& d_{31}=\frac{C_{22} e_{31}-C_{12} e_{32}}{C_{11} C_{22}-C_{12}^{2}} \\
& d_{32}=\frac{C_{11} e_{32}-C_{12} e_{31}}{C_{11} C_{22}-C_{12}^{2}}
\end{aligned}
$$

Fortunately, the CCB monolayer also possesses only outof-plane piezoelectric effect. These also imply that the piezoelectric effects of CCB monolayer can be induced with a uniaxial strain being applied along $\mathrm{x}$ or/and $\mathrm{y}$ direction, which is different monolayer CCC and CBB with shear strain.

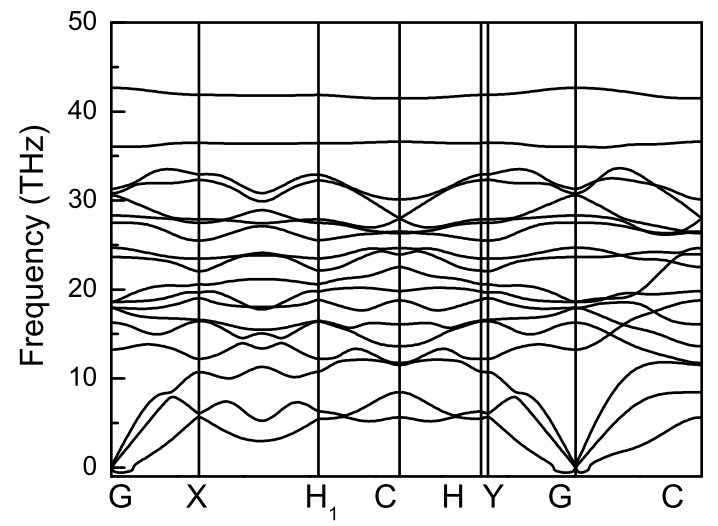

FIG. 4. The phonon band dispersion of CCB monolayer.

Compared with tetragonal structure of $\mathrm{CCC}$ and $\mathrm{CBB}$, the crystal structure of CCB changes into orthorhombic, and the optimized lattice constants $a=b=3.771 \AA$ and $\gamma=92.107$. To confirm the dynamic stability of CCB monolayer, the phonon spectrum are calculated by VASP + Phonopy code with a supercell of $4 \times 4 \times 1$ using the finite displacement $\operatorname{method}^{30}$. Figure 4 shows the calculated phonon spectrum. Although there are some negligibly small imaginary frequencies near the $\mathrm{G}$ point due to calculation error, no imaginary frequencies in the other q points throughout the Brillouin zone are observed, which implies the dynamic stability of CCB monolayer. The mechanical stability of CCB monolayer can be examined by elastic constants $C_{i j}$. The calculated $C_{11}=142.36 \mathrm{Nm}^{-1}, C_{22}=193.07 \mathrm{Nm}^{-1}, C_{12}=34.40$ $\mathrm{Nm}^{-1}$ and $C_{66}=61.71 \mathrm{Nm}^{-1}$, which satisfy the Born criteria of mechanical stability ${ }^{28,29}$. The Young's modulus $C_{2 D}(\theta)$ and Poisson's ratio $\nu(\theta)$ as a function of in-plane $\theta$ can be attained on the basis of the elastic constants, as follows ${ }^{28}$ :

$$
\begin{gathered}
C_{2 D}(\theta)=\frac{C_{11} C_{22}-C_{12}^{2}}{C_{11} \sin ^{4} \theta+A \sin ^{2} \theta \cos ^{2} \theta+C_{22} \cos ^{4} \theta} \\
\nu(\theta)=\frac{C_{12} \sin ^{4} \theta-B \sin ^{2} \theta \cos ^{2} \theta+C_{12} \cos ^{4} \theta}{C_{11} \sin ^{4} \theta+A \sin ^{2} \theta \cos ^{2} \theta+C_{22} \cos ^{4} \theta}
\end{gathered}
$$

In which $A=\left(C_{11} C_{22}-C_{12}^{2}\right) / C_{66}-2 C_{12}$ and $B=$ $C_{11}+C_{22}-\left(C_{11} C_{22}-C_{12}^{2}\right) / C_{66}$. We show the calculated $C_{2 D}(\theta)$ and $\nu(\theta)$ in Figure 5 . Both the Young's modulus $C_{2 D}(\theta)$ and Poisson's ratio $\nu(\theta)$ show mechanical anisotropy. According to calculated $C_{2 D}(\theta), \mathrm{CCB}$ monolayer is softer along the $\mathrm{x}$ than $\mathrm{y}$ direction, which is due to $\mathrm{B}-\mathrm{B}$ bond along $\mathrm{x}$ direction and $\mathrm{C}-\mathrm{C}$ bond along $\mathrm{y}$ direction. A high Young's modulus implies that the material is rigid, and the calculated results show that strain can easily tune it's physical properties along $\mathrm{x}$ direction. It is found that the Poisson's ratio $\nu$ along $\mathrm{x}$ direction (0.179) is smaller than one along y direction (0.242).

A 2D material with piezoelectricity not only should break inversion symmetry, but also has a band gap. The energy band structures of CCB along with $\mathrm{CCC}$ and $\mathrm{CBB}$ 

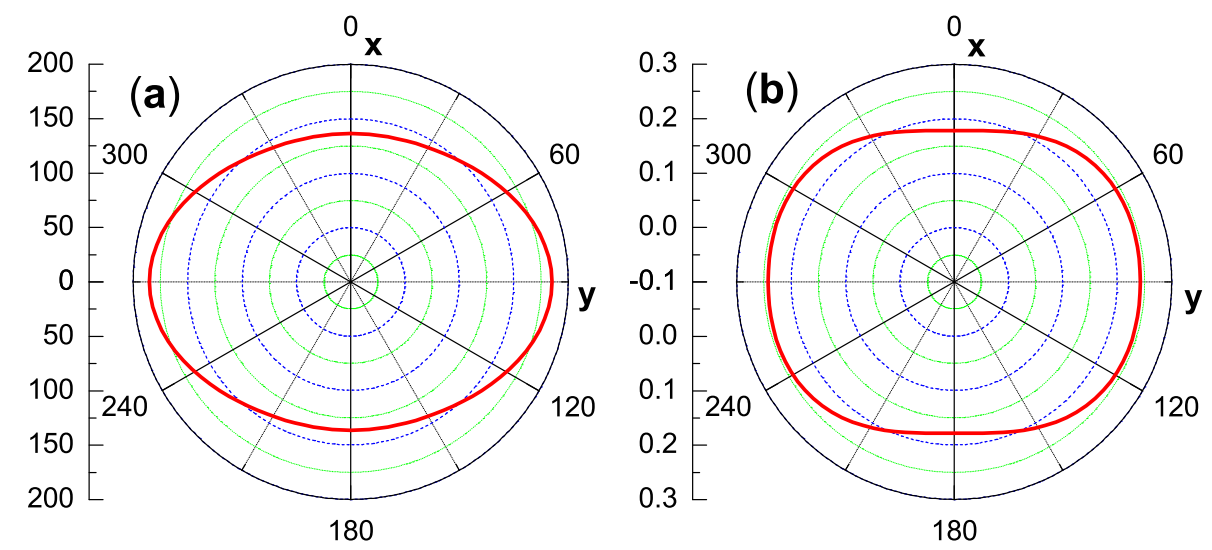

FIG. 5. (Color online)The Young's modulus and Possion's ratio of CCB monolayer as a function of the angle $\theta$.
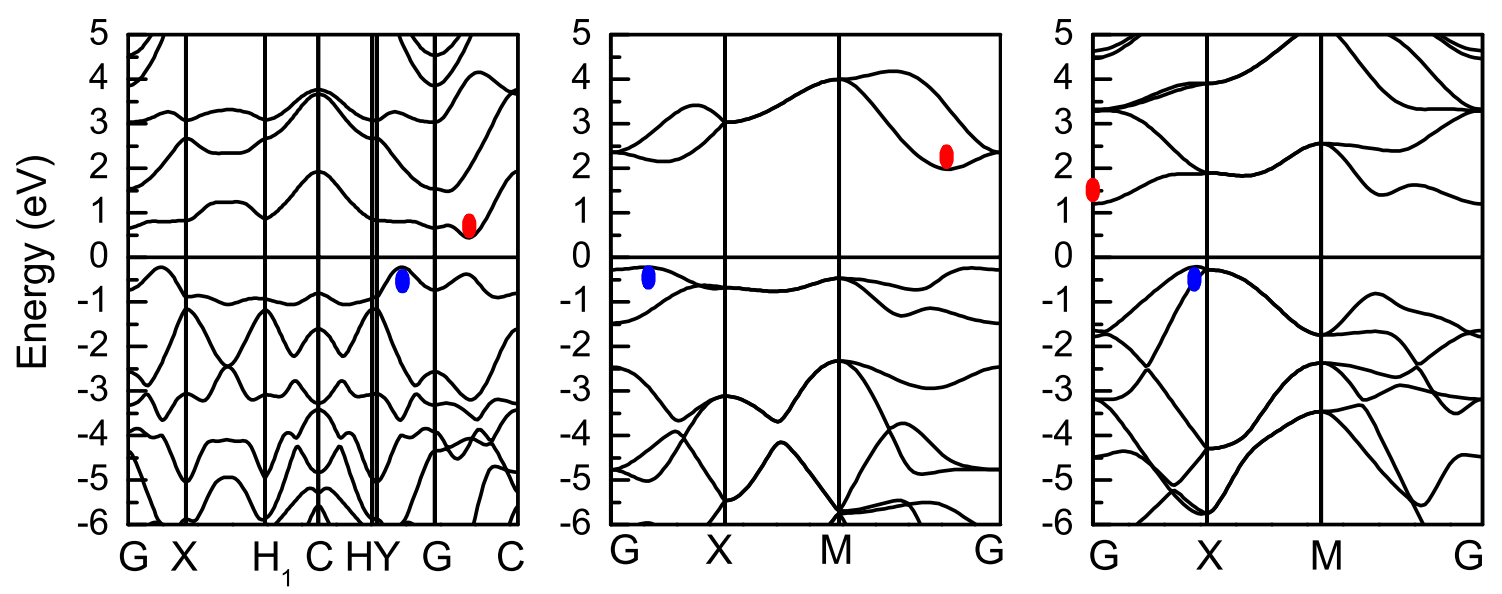

FIG. 6. (Color online)The energy band structures of monolayer CCB (Left), CCC (Middle) and CBB (Right) using GGA, and the VBM and CBM are marked by blue and red ellipses, respectively.

are plotted in Figure 6. It is clearly seen that CCB is an indirect band-gap semiconductor with a band gap of 0.66 $\mathrm{eV}$, which is smaller than indirect gap $2.20 \mathrm{eV}$ of $\mathrm{CCC}$ or $1.41 \mathrm{eV}$ of CBB. The valance band maximum (VBM) lies on the G-Y path, while the conduction band minimum $(\mathrm{CBM})$ is located on the G-C path. In fact, the valance band extrema (VBE) along G-X path is very close to VBM due to $\gamma$ being very close to 90 , and the difference is less than $1 \mathrm{meV}$. The carrier mobility of a semiconductors is an important factor for the application of electron device. The carrier mobility of a $2 \mathrm{D}$ material $\left(\mu_{2 D}\right)$ by the deformation potential (DP) theory proposed by Bardeen and Shockley ${ }^{31}$ is defined as:

$$
\mu_{2 D}=\frac{e \hbar^{3} C_{2 D}}{K_{B} T m^{*} m_{d} E_{l}^{2}}
$$

where $T, m^{*}$ and $m_{d}\left(\sqrt{m_{x} m_{y}}\right)$ are the temperature, the effective mass in the transport direction and the average effective mass. The $C_{2 D}$ is the Young's modulus derived from elastic constants $C_{i j}$. In addition, $E_{l}$ is the DP constant defined by $E_{l}=\Delta E / \delta\left(\delta=\Delta l / l_{0}\right)$, where $\Delta E$ is the energy shift of the band edge of CBM or VBM with respect to the vacuum level after applying uniaxial strain. After attaining $\mu_{2 D}$, the relaxation time $\tau$ can be attained by:

$$
\tau=\mu_{2 D} m^{*} / e
$$

According to DP theory, the rectangular supercell is used to calculate carrier mobilities of monolayer CCB along the $\mathrm{x}$ and $\mathrm{y}$ directions in Figure 3. The calculated effective masses for electrons (CBM) and holes (VBM) with GGA are shown in Table III. The band energies of the VBM and CBM with respect to the vacuum energy as a function of $\Delta x / x$ and $\Delta y / y$ are plotted in Figure 7 , and the DP constant $E_{l}$ is calculated by linearly fitting these data. The carrier mobility and relaxation time for the electrons and holes of monolayer $\mathrm{CCB}$ on the basis of the calculated effective mass, elastic constant, and deformation potential constant are calculated along $\mathrm{x}$ and $\mathrm{y}$ directions at $300 \mathrm{~K}$, which are summarized in Table III. It is found that the electron mobility along y direction $\left(8865.23 \mathrm{~cm}^{2} \mathrm{~V}^{-1} \mathrm{~s}^{-1}\right)$ is almost 97 times larger than that along $\mathrm{x}$ direction $(91.92$ $\left.\mathrm{cm}^{2} \mathrm{~V}^{-1} \mathrm{~s}^{-1}\right)$. However, the hole mobilities along both 
TABLE III. For CCB monolayer, elastic modulus $\left(C_{2 D}\right)$, effective mass $\left(m^{*}\right)$, deformation potential $\left(E_{l}\right)$, carrier mobility $\left(\mu_{2 D}\right)$ and relaxation time $(\tau)$ at $300 \mathrm{~K}$.

\begin{tabular}{ccccccc}
\hline \hline Carrier type & & $C_{2 D}\left(\mathrm{Nm}^{-1}\right)$ & $m^{*}$ & $E_{l}(\mathrm{eV})$ & $\mu_{2 D}\left(\mathrm{~cm}^{2} \mathrm{~V}^{-1} \mathrm{~s}^{-1}\right)$ & \multicolumn{1}{c}{$\tau$} \\
\hline \hline Electrons & $\mathrm{x}$ & 136.23 & 1.57 & 5.00 & 91.92 & $8.18 \times 10^{-14}$ \\
& $\mathrm{y}$ & 184.76 & 0.42 & -1.15 & 8865.23 & $2.10 \times 10^{-12}$ \\
\multirow{2}{*}{ Holes } & $\mathrm{x}$ & 136.23 & -0.45 & -9.20 & 114.63 & $2.90 \times 10^{-14}$ \\
& $\mathrm{y}$ & 184.76 & -1.02 & 6.97 & 118.75 & $7.46 \times 10^{-14}$ \\
\hline \hline
\end{tabular}
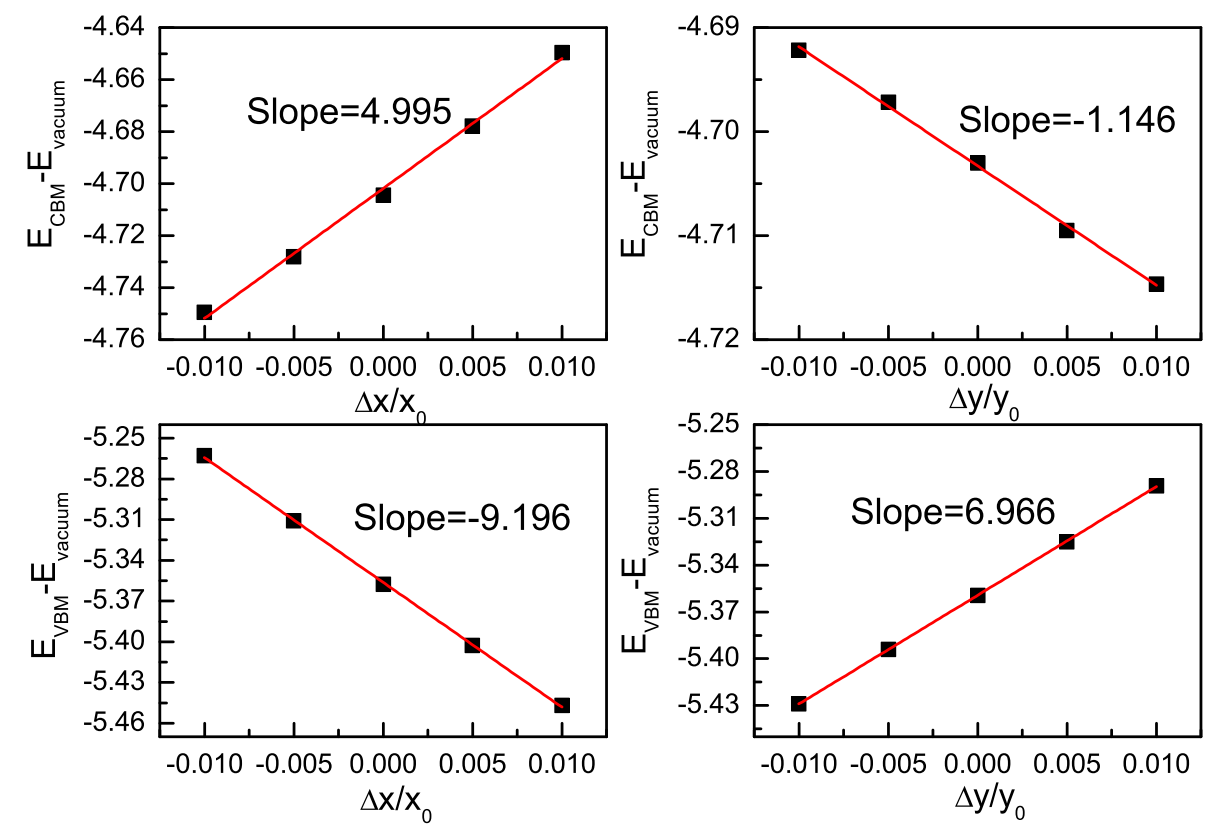

FIG. 7. (Color online)With respect to the vacuum energy, the band energies of the VBM and CBM of CCB monolayer as a function of lattice dilation along both $\mathrm{x}$ and $\mathrm{y}$ directions. The red solid lines are linear fitting curves, and the fitted slopes are given, corresponding to the DP.

$\mathrm{x}\left(114.63 \mathrm{~cm}^{2} \mathrm{~V}^{-1} \mathrm{~s}^{-1}\right)$ and $\mathrm{y}\left(118.75 \mathrm{~cm}^{2} \mathrm{~V}^{-1} \mathrm{~s}^{-1}\right)$ directions are very low, which is close to electron mobility along $\mathrm{x}$ direction.

In fact, the CCB is a representative Janus monomlayer. The asymmetric along the $\mathrm{z}$ direction with respect to central $\mathrm{C}$ atomic layer results in an out-of-plane piezoelectricity. However, mirror symmetry along $\mathrm{x}$ and $\mathrm{y}$ directions gives rise to disappeared in-plane piezoelectricity. This means that an in-plane stress or strain can only induce a polarization change vertical to the plane. Based on calculated $C_{i j}$ and $e_{i j}$, the out-of-plane piezoelectric strain coefficient $d_{31}$ and $d_{32}$ can be attained, and the corresponding values are $-0.505 \mathrm{pm} / \mathrm{V}$ and $0.273 \mathrm{pm} / \mathrm{V}$, respectively. The out-of-plane $d_{31}$ is obviously higher than out-of-plane ones of many other $2 \mathrm{D}$ materials, such as functionalized h-BN $(0.13 \mathrm{pm} / \mathrm{V})^{32}$, MoSSe $(0.29 \mathrm{pm} / \mathrm{V})^{33}$, SbTeI (-0.37 pm/V) ${ }^{34}$, MoSTe $(0.4 \mathrm{pm} / \mathrm{V})^{35}$, Janus group-III materials $(0.46 \mathrm{pm} / \mathrm{V})^{36}$ and $\alpha-\operatorname{In}_{2} \mathrm{Se}_{3}(0.415 \mathrm{pm} / \mathrm{V})^{37}$. It is lower than one of BiTeI (-0.66 pm/V) $)^{34}, \mathrm{Sc}_{2} \mathrm{CO}_{2}(0.78 \mathrm{pm} / \mathrm{V})^{38}$ or $\mathrm{La}_{2} \mathrm{CO}_{2}$ $(0.65 \mathrm{pm} / \mathrm{V})^{38}$. However, the monolayer BiTeI, $\mathrm{Sc}_{2} \mathrm{CO}_{2}$ or $\mathrm{La}_{2} \mathrm{CO}_{2}$ not only has out-of-plane piezoelectricity, but also has in-plane piezoelectricity. Some related data are summarized in Table II. Although the $d_{36}$ (CCC monolayer) and $d_{31}$ or $d_{32}$ (CCB monolayer) are all related to the out-of-plane piezoelectric effects, they are obviously different. The $d_{36}$ represents the piezoelectric response between the out-of-plane polarization and the in-plane shearing deformation, while $d_{31}$ or $d_{32}$ is referred to as the coupling between the out-of-plane polarization and the in-plane normal strain.

\section{CONCLUSION}

In summary, the related piezoelectric effects of CCC monolayer are studied by using reliable first-principles calculations. Due to special symmetry for CCC monolayer, only out-of-plane $d_{36}$ exists, but it is very small. Firstly, we use both biaxial and uniaxial strains to tune piezoelectric properties of CCC monolayer. At $-2 \%$ biaxial (-4\% uniaxial) strain, the $d_{36}$ is increased only by $3.1 \%(13.9 \%)$, and the enhancement is very small. Inspiring from the already synthesized MoSSe monolayer, 
a Janus CCB monolayer with dynamic and mechanical stability is constructed, and the asymmetric along the $\mathrm{z}$ direction and mirror symmetry along $\mathrm{x}$ and $\mathrm{y}$ directions induce pure out-of-plane piezoelectricity. It is also found that $\mathrm{CCB}$ monolayer is a semiconductor, which is necessary for piezoelectric application. The calculated $d_{31}$ and $d_{32}$ are $-0.505 \mathrm{pm} / \mathrm{V}$ and $0.273 \mathrm{pm} / \mathrm{V}$, respectively, and the out-of-plane piezoelectric effect are obviously improved, compared with $d_{36}$ of CCC monolayer. The out-of-plane piezoelectricity $d_{31}$ of CCB monolayer is higher compared with other many $2 \mathrm{D}$ known materials. The very high room-temperature electronic mobility $\left(8865.23 \mathrm{~cm}^{2} \mathrm{~V}^{-1} \mathrm{~s}^{-1}\right)$ along $\mathrm{y}$ direction is predicted, which is very higher than that of Si (about 1400 $\left.\mathrm{cm}^{2} \mathrm{~V}^{-1} \mathrm{~s}^{-1}\right)$. Our works not only supply an experimental proposal for achieving large pure out-of-plane piezoelectric effect, but also offer an insight into piezoelectric effect of penta-graphene.

\section{ACKNOWLEDGMENTS}

This work is supported by the Natural Science Foundation of Shaanxi Provincial Department of Education (19JK0809). We are grateful to the Advanced Analysis and Computation Center of China University of Mining and Technology (CUMT) for the award of CPU hours and WIEN2k/VASP software to accomplish this work.
1 S. Zhang, J. Zhou, Q. Wang, X. Chen, Y. Kawazoe and P. Jena, Proc. Natl. Acad. Sci. Unit. States Am. 112, 2372 (2015).

2 F. Li, K. Tu, H. Zhang and Z. Chen, Phys. Chem. Chem. Phys. 17, 24151 (2015).

3 Y. Ma, L. Kou, X. Li, Y. Dai and T. Heine, NPG Asia Mater. 8, e264 (2016).

4 J. Li, X. Fan, Y. Wei, H. Liu, S. Li, P. Zhao and G. Chen, Sci. Rep. 6, 33060 (2016).

5 H. Sun, S. Mukherjee and C. V. Singh, Phys. Chem. Chem. Phys. 18, 26736 (2016).

${ }^{6}$ F. Q. Wang, J. Yu, Q. Wang, Y. Kawazoe and P. Jena, Carbon 105, 424 (2016).

7 W. Xu, G. Zhang and B. Li, J. Chem. Phys. 143, 154703 (2015).

8 J. Sun, Y. G. Guo, Q. Wang and Y. Kawazoe, Carbon 145, 445 (2019).

${ }^{9}$ L. L. Liu, Y. Wang, C. P. Chen, H. X. Yu, L. S. Zhao and X. C. Wang, RSC Adv. 7, 40200 (2017).

${ }^{10}$ W. Wu and Z. L. Wang, Nat. Rev. Mater. 1, 16031 (2016).

11 W. Wu, L. Wang, Y. Li, F. Zhang, L. Lin, S. Niu, D. Chenet, X. Zhang, Y. Hao, T. F. Heinz, J. Hone and Z. L. Wang, Nature 514, 470 (2014).

${ }^{12}$ H. Zhu, Y. Wang, J. Xiao, M. Liu, S. Xiong, Z. J. Wong, Z. Ye, Y. Ye, X. Yin and X. Zhang, Nat. Nanotechnol. 10, 151 (2015).

13 A. Y. Lu, H. Zhu, J. Xiao, C. P. Chuu, Y. Han, M. H. Chiu, C. C. Cheng, C. W. Yang, K. H. Wei, Y. Yang, Y. Wang, D. Sokaras, D. Nordlund, P. Yang, D. A. Muller, M. Y. Chou, X. Zhang and L. J. Li, Nat. Nanotechnol. 12, 744 (2017).

14 L. Dong, J. Lou and V. B. Shenoy, ACS Nano, 11, 8242 (2017).

15 M. N. Blonsky, H. L. Zhuang, A. K. Singh and R. G. Hennig, ACS Nano, 9, 9885 (2015).

16 R. X. Fei, We. B. Li, J. Li and L. Yang, Appl. Phys. Lett. 107, 173104 (2015)

17 K. N. Duerloo, M. T. Ong and E. J. Reed, J. Phys. Chem. Lett. 3, 2871 (2012).

18 Y. Chen, J. Y. Liu, J. B. Yu, Y. G. Guo and Q. Sun, Phys. Chem. Chem. Phys. 21, 1207 (2019).
19 X. Wu, D. Vanderbilt and D. R. Hamann, Phys. Rev. B 72, 035105 (2005).

20 P. Hohenberg and W. Kohn, Phys. Rev. 136, B864 (1964); W. Kohn and L. J. Sham, Phys. Rev. 140, A1133 (1965).

21 G. Kresse, J. Non-Cryst. Solids 193, 222 (1995).

22 G. Kresse and J. Furthmüller, Comput. Mater. Sci. 6, 15 (1996).

23 G. Kresse and D. Joubert, Phys. Rev. B 59, 1758 (1999).

24 J. P. Perdew, K. Burke and M. Ernzerhof, Phys. Rev. Lett. 77, 3865 (1996).

25 N. Jena, Dimple, S. D. Behere and A. D. Sarkar, J. Phys. Chem. C 121, 9181 (2017).

26 S. D. Guo, X. S. Guo, Y. Y. Zhang and K. Luo, arXiv:1910.08700 (2019).

27 Dimple, N. Jena, A. Rawat, R. Ahammed, M. K. Mohanta and A. D. Sarkar, J. Mater. Chem. A 6, 24885 (2018).

28 E. Cadelano, P. L. Palla, S. Giordano and L. Colombo, Phys. Rev. B 82, 235414 (2010).

29 R. C. Andrew, R. E. Mapasha, A. M. Ukpong and N. Chetty, Phys. Rev. B 85, 125428 (2012).

30 A. Togo, F. Oba, and I. Tanaka, Phys. Rev. B 78, 134106 (2008).

31 S. Bruzzone and G. Fiori, Appl. Phys. Lett. 99, 222108 (2011).

32 A. A. M. Noor, H. J. Kim and Y. H. Shin, Phys. Chem. Chem. Phys. 16, 6575 (2014).

33 S. D. Guo, X. S. Guo, R. Y. Han and Y. Deng, Phys. Chem. Chem. Phys. (2019). DOI: 10.1039/C9CP04590B.

34 S. D. Guo, X. S. Guo, Z. Y. Liu, Y. N. Quan, arXiv:1909.13227 (2019).

35 M. Yagmurcukardes, C. Sevik and F. M. Peeters, Phys. Rev. B 100, 045415 (2019).

36 Y. Guo, S. Zhou, Y. Z. Bai, J. J. Zhao, Appl. Phys. Lett. 110, 163102 (2017).

37 L. Hu and X. R. Huang, RSC Adv. 7, 55034 (2017).

38 J. Tan, Y. H. Wang, Z. T. Wang et al., Nano Energy 65, 104058 (2019). 